\title{
Comparative Efficacy and Toxicity of Different Species of Sargassum in Haizao Yuhu Decoction in PTU-Induced Goiter Rats
}

\author{
Linlin Xiu, Gansheng Zhong, Dianna Liu, Shaohong Chen, Haiyan Liu, and Feng Chen
}

Beijing University of Chinese Medicine, Beijing, China

Correspondence should be addressed to Gansheng Zhong; 1206284369@qq.com

Received 18 November 2016; Accepted 18 May 2017; Published 21 June 2017

Academic Editor: Shruti Shukla

Copyright (C) 2017 Linlin Xiu et al. This is an open access article distributed under the Creative Commons Attribution License, which permits unrestricted use, distribution, and reproduction in any medium, provided the original work is properly cited.

\begin{abstract}
Background. Haizao Yuhu Decoction has been widely used to treat thyroid-related diseases especially goiter with few side effects in traditional Chinese medicine (TCM), including herb pair Sargassum (HZ) and Glycyrrhizae Radix et Rhizoma (GC), as one of "eighteen antagonistic medicaments." The two different species of Sargassum, Sargassum fusiforme (Sf) and Sargassum pallidum (Sp), are not clearly differentiated in clinical use, so that herb pair Sf-GC and Sp-GC could show different effect and toxicity. Methods. We investigated the antigoitrous effect and toxicity and clarified the potential underlying mechanism of the two different species of Sargassum in HYD (HYDf and HYDp) in PTU-reduced goiter rats. Results. The results demonstrated that both HYDf and HYDp could exhibit antigoitrous effect through alterations in hypothalamus-pituitary-thyroid (HPT) axis and inhibition of the TPO gene expression; there is no difference in the antigoitrous effects between the two different species of Sargassum application in HYD. Conclusion. This study evaluated the safety and efficacy of herb pair HZ-GC applied in HYD in goiter rats at molecular, cellular, and whole level and compared the two species of Sargassum further. We provide a reliable way to clarify the possible mechanism of the antagonistic medicament herb pair HZ-GC for its application.
\end{abstract}

\section{Introduction}

Goiter, an enlargement of the thyroid gland, is a common problem in clinical practice [1]. It can be associated with a number of thyroid diseases such as thyroid dysfunction (hyperthyroidism and hypothyroidism), autoimmune thyroid disease (Graves' disease and Hashimoto's thyroiditis) [2, $3]$, thyroiditis, and thyroid cancer $[4,5]$. Regardless of varies clinical manifestation, the etiology of goiter is essentially the same: the thyroid attempting to adapt to circumstances of changes in the thyroid's ability to secrete adequate amounts of hormones [1], of which hypothyroidism is the most common in recent decade [6]. Hypothyroidism, abnormalities in thyroid hormone synthesis or thyroid dyshormonogenesis, is a common endocrine disorder [7] involved with multiple organs. The single most common cause of hypothyroidism worldwide was undoubtedly iodine deficiency. Where it is not a problem, the most common causes are chronic autoimmune thyroiditis and previous thyroid surgery or ${ }^{131} \mathrm{I}$ therapy.
Its clinical characteristics are complex. Internationally, conventional drugs, such as potassium iodide and levothyroxine, are recommended for patients with hypothyroidism. However, their respective curative effects remain controversial [8].

According to traditional Chinese medicine (TCM) theory, goiter is mainly due to "Qi" stagnation and "phlegm" stasis [9]. Haizao Yuhu Decoction (HYD), which is described in a famous TCM monograph Waike Zhengzong (Summary of Surgical Medicine) in Ming Dynasty of China, has been used for approximately 500 years and famous for its efficacy in treating thyroid-related diseases especially goiter based on the clinical study [10]. It is worthwhile to mention that there is a herb pair Sargassum (Haizao, HZ) and Glycyrrhizae Radix et Rhizoma (Gancao, GC), one of the so-called "eighteen antagonistic medicaments" in TCM documents, indicating that the two herbs should not be applied simultaneously, whereas the herb pair HZ-GC has been widely used in HYD for the treatment of thyroid dysfunction, galactophorous hyperplasia, and ovarian cyst [11]. In recent decades, some 
TABLE 1: Compositions of HYD.

\begin{tabular}{|c|c|c|c|}
\hline \multirow{2}{*}{$\begin{array}{l}\text { HYDf/HYDp } \\
\text { Latin name }\end{array}$} & \multicolumn{3}{|c|}{ 海藻玉壶汤羊栖菜组/海藻玉壶汤海蒿子组 } \\
\hline & Chinese name & Source & Daily adult dose (g) \\
\hline Glycyrrhiza uralensis Fisch. & 生甘草 & Root and rhizome & 24 \\
\hline $\begin{array}{l}\text { Sargassum fusiforme (Harv.) Setch. } \\
\text { /Sargassum pallidum (Turn.) C.Ag. }\end{array}$ & $\begin{array}{l}\text { 羊栖菜 } \\
\text { /海蒿子 }\end{array}$ & Frond & 24 \\
\hline Pinellia ternata (Thunb.) Breit. & 法半夏 & Tuber & 9 \\
\hline Fritillaria thunbergii Miq. & 浙贝母 & Bulb & 9 \\
\hline Laminaria japonica Aresch. & 海带 & Thallus & 9 \\
\hline Forsythia suspensa (Thunb.) Vahl. & 连翘 & Fruit & 9 \\
\hline Ligusticum chuanxiong Hort. & 川芎 & Rhizome & 9 \\
\hline Angelica pubescens Maxim. f. biserrata Shan et Yuan & 独活 & Root & 9 \\
\hline Laminaria japonica Aresch./Ecklonia kurome Okam. & 昆布 & Thallus & 9 \\
\hline Citrus reticulata Blanco & 青皮 & Fruitlet & 9 \\
\hline Citrus reticulata Blanco & 陈皮 & Pericarp & 9 \\
\hline Angelica sinensis (Oliv.) Diels. & 当归 & Root & 9 \\
\hline
\end{tabular}

literature research, clinical study, and animal experiment have been carried out to evaluate the efficacy and safety of the combination $[12,13]$. However, their respective curative effects and mechanisms remain controversial and there is limited scientific evidence to establish the safety and efficacy of these herbal products.

Additionally, Sargassum fusiforme (Yangqicai, Sf) and Sargassum pallidum (Haihaozi, Sp) are two different species of Sargassum officially recorded in the Chinese Pharmacopoeia but not clearly differentiated in clinical use. The pharmacological effects of them have been extensively studied [14], but there are few comparative studies between them.

What roles do these two different species of Sargassum play in the herb pair HZ-GC, and whether the different species are related to impact effects of HYD? Accordingly, we proposed the present study to systematically evaluate antigoitrous effect and toxicity and to clarify the underlying mechanism of the two different species of Sargassum in HYD in PTU-reduced goiter rats at molecular, cellular, and whole level.

\section{Materials and Methods}

2.1. Preparation of Haizao Yuhu Decoction. HYD is a decoction of 12 Chinese herbs as listed in Table 1.

As there are two species of Sargassum, Haizao Yuhu Decoction with the species of Sargassum fusiforme (HYDf) and Haizao Yuhu Decoction with the species of Sargassum pallidum (HYDp) were prepared separately. The rat equivalent doses were selected based on the clinical adult dosage provided by the Chinese Pharmacopoeia and calculated according to the ratio of body-surface area between human and rat.

All herbs used in the study were purchased from Beijing Shuangqiao Co., Ltd. (Beijing, China) and Anhui Tongling Co., Ltd. (Tongling, China) and authenticated in accordance with the standard of the Chinese Pharmacopoeia by Professor Li (School of Basic Medical Science, Beijing University of
Chinese Medicine). The mixtures were soaked in distilled water in a ratio of $1 / 10(\mathrm{~g} / \mathrm{ml})$ for $2 \mathrm{~h}$ and then subsequently boiled for a further $60 \mathrm{~min}$. After saving the supernatant collected by filtering, the residue was added with a ratio of $1 / 8(\mathrm{~g} / \mathrm{ml})$ distilled water and boiled again for a further $30 \mathrm{~min}$. Finally, the two portions of aqueous extracts were pooled together and concentrated to a density of $1.0 \mathrm{~g} / \mathrm{ml}$ crude herb by means of heating evaporation.

2.2. Experimental Reagents. Propylthiouracil (PTU) was purchased from Zhaohui Pharmaceutical Technology Corporation (Shanghai, China); Euthyrox was purchased from MerkKGaA (Darmstadt, Germany).

2.3. Preparation of Goiter Model and Grouping. 50 Male and female Wistar rats (180-220 g) were purchased from Charles River Laboratories, Beijing, China (Certificate of Conformity: SCXK (Beijing) 2012-0001). The animals had free access to drinking water and regular chow with a standard commercial rat feed every day and were housed in a room under controlled conditions (temperature $22 \pm 2^{\circ} \mathrm{C}$, humidity $50 \pm 10 \%$, and light illumination $12 \mathrm{~h} / \mathrm{d}$ ) (Certificate of Conformity: SCXK (Beijing) 2011-0024). Experimental protocols were approved by the Experimental Animal Care and Ethics Committees of Beijing University of Chinese Medicine.

After 3 days of acclimatization, the rats were randomly divided into 5 groups: normal, model, Euthyrox, HYDf, and HYDp groups; all rats except those in the normal control group received intragastric administration of PTU at a dosage of $0.01 \mathrm{~g} / \mathrm{kg}$ daily for 14 days to induce the goitrous model according to previously reports [15] and were given different treatments for 28 days, as shown in Table 2.

2.4. Sample Collection. After 28 days of treatment, diets were removed from the cages $12 \mathrm{~h}$ before the animals were sacrificed. Blood samples were collected and centrifuged at $3000 \times \mathrm{g}$ for $12 \mathrm{~min}$ to obtain serum. 
TABLE 2: Experimental schema.

\begin{tabular}{lcc}
\hline Groups & Dose & Treatment \\
\hline Normal & $10 \mathrm{ml} / \mathrm{kg} / \mathrm{d}$ & Rats received only normal feeds, distilled water, and gavage normal saline $(1 \mathrm{ml} / 100 \mathrm{~g}$ body weight $/$ day $)$ \\
Model & $0.01 \mathrm{~g} / \mathrm{kg} / \mathrm{ml}$ & Rats received PTU every two days, distilled water, and gavage distilled water $(1 \mathrm{ml} / 100 \mathrm{~g} /$ day $)$ \\
Euthyrox & $0.01 \mathrm{~g} / \mathrm{kg} / \mathrm{ml}$ & Rats received Euthyrox and distilled water \\
HYDf & $12.06 \mathrm{~g} / \mathrm{kg} / \mathrm{d}$ & Rats received HYDf $(1 \mathrm{ml} / 100 \mathrm{~g} /$ day $)$ \\
HYDp & $12.06 \mathrm{~g} / \mathrm{kg} / \mathrm{d}$ & Rats received HYDp $(1 \mathrm{ml} / 100 \mathrm{~g} /$ day $)$ \\
\hline
\end{tabular}

Thyroid glands, liver, and kidney were isolated, weighed, and stored at $-80^{\circ} \mathrm{C}$ for pathological observation.

2.5. General Observations. Clinical signs and mortality were recorded twice a day (before and after treatment) throughout the study period. The body weight (BW) and rectal temperature of each rat were measured at the initiation of treatment and once a week during the study period.

\subsection{Thyroid Function Assessment, Relative Organ Weights} Assessment, and Biochemical Analysis. Absolute organ (thyroid gland, liver, and kidney) weights were measured and relative organ weights (organ-to-body weight ratios, g/100 g BW) were calculated.

The levels of serum free triiodothyronine (FT3), free thyroxine (FT4), thyroid stimulating hormone (TSH), and thyrotropin releasing hormone (TRH) were determined by radioimmunoassay using commercially available kits (purchased from Biosino. Inc., Beijing, China) according to manufacturer's instructions.

Serum alanine aminotransferase (ALT), aspartate aminotransferase (AST), alkaline phosphatase (ALP), blood urea nitrogen (BUN), creatinine (Cr), and uric acid (UA) were determined to assess liver and kidney function using commercially available kits (purchased from Biosino. Inc., Beijing, China) according to manufacturer's instructions.

2.7. Histopathology. After all animals had been sacrificed, thyroid glands were removed and divided into two parts; one was put in a buffer solution of $10 \%$ formalin and embedded in paraffin. Six to ten $4 \mu \mathrm{m}$-thick sections were prepared in a noncontiguous way and dyed with hematoxylin-eosin (H-E); the stained areas were viewed using an optical microscope to assess the pathologic alterations of thyroid tissues in different groups and to evaluate the effects of the treatments.

Another one was immediately stored in liquid nitrogen at $-80^{\circ} \mathrm{C}$ for real-time reverse transcription-polymerase chain reaction (RT-PCR) analysis.

2.8. RT-PCR Analysis. Thyroglobulin (Tg) mRNA and thyroid peroxidase (TPO) mRNA expression were determined using RT-PCR. Total RNA was extracted from thyroid glands using the Trizol reagent (Invitrogen, USA). RNA yields and purity were assessed by spectrophotometric analysis (Spectronic Unicam, USA). Total RNA $(1 \mu \mathrm{g} / \mu \mathrm{l})$ transcription was performed using an in vitro transcription Kit (PrimeScript RT reagent Kit Perfect Real Time, TaKaRa, Japan). RT-PCR reactions were performed with $20 \mu \mathrm{l}$ reactions that consisted
TABLE 3: The primer sequences.

\begin{tabular}{lcc}
\hline Primer & \multicolumn{1}{c}{ Sequence $\left(5^{\prime}\right.$ to $\left.3^{\prime}\right)$} & Length \\
\hline \multirow{2}{*}{ TPO } & FW: CTCCACGGATGCACTATCAG & $180 \mathrm{bp}$ \\
& RV: TTCTACCGA CGGAGGACAGA & \\
\multirow{2}{*}{ Tg } & FW: GTGAACGCCTCTGTGACAGA & $280 \mathrm{bp}$ \\
& RV: ACGAAACCTGAGGACCGTCT & \\
GAPDH & FW: TTCACCACCATGGAGAAGGC & \\
& RV: ACTGTACGGCGGACCTCTTT & $430 \mathrm{bp}$ \\
\hline
\end{tabular}

of $10 \mu \mathrm{l} 2 \mathrm{x}$ Taq PCR MasterMix (Solalbio, Beijing, China), $0.5 \mu \mathrm{l}$ PCR forward primer $(10 \mu \mathrm{M}), 0.5 \mu \mathrm{l}$ PCR reverse primer $(10 \mu \mathrm{M}), 3 \mu \mathrm{l} \mathrm{cDNA}, 0.5 \mu \mathrm{l}$ GAPDH forward primer, $0.5 \mu \mathrm{l} \mathrm{GAPDH}$ reverse primer, and $5 \mu$ l double-distilled water. The PCR cycling conditions comprised a denaturation step for $3 \mathrm{~min}$ at $94^{\circ} \mathrm{C}$, followed by 34 cycles of denaturation $\left(94^{\circ} \mathrm{C}\right.$ for $\left.30 \mathrm{~s}\right)$, annealing $\left(65^{\circ} \mathrm{C}\right.$ for $\mathrm{TPO}, 62^{\circ} \mathrm{C}$ for $\mathrm{Tg}$ and $56^{\circ} \mathrm{C}$ for $\mathrm{GAPDH}$ for $\left.30 \mathrm{~s}\right)$, and extension $\left(72^{\circ} \mathrm{C}\right.$ for $\left.1 \mathrm{~min}\right)$. After the last cycle, all PCR products were subjected to a final extension for $8 \mathrm{~min}$ at $72^{\circ} \mathrm{C}$. The primer sequences were shown in Table 3.

PCR products were combined and then electrophoresed on $1.5 \%$ agarose gels containing ethidium bromide; data analysis was carried out using Alpha Ease FC software (Alpha Intech, USA). Values obtained for Tg and TPO were normalized against values obtained for GAPDH, and the results were expressed as relative integrated intensity.

2.9. Statistical Analysis. The data was analyzed using the SPSS 21.0 software and expressed as the mean \pm standard deviation (SD). Differences between groups were examined for statistical significance using one-way analysis of variance (ANOVA; SPSS 20.0 for Windows; SPSS Inc., USA). The results were considered statistically significant when $P<$ 0.05 .

\section{Results}

3.1. Body Weight and Temperature Changes in PTU-Induced Goiter Rats. Body weight of rats was increased in a timedependent manner (Figure 1(a)). PTU treatment for 14 days resulted in marked decreases in body weight and rectal temperature (Figure 1).

There were statistical increases of body weight in Euthyrox, HYDf, and HYDp treatment groups compared to the model group after 28 days drug treatment $(P<0.05)$. 


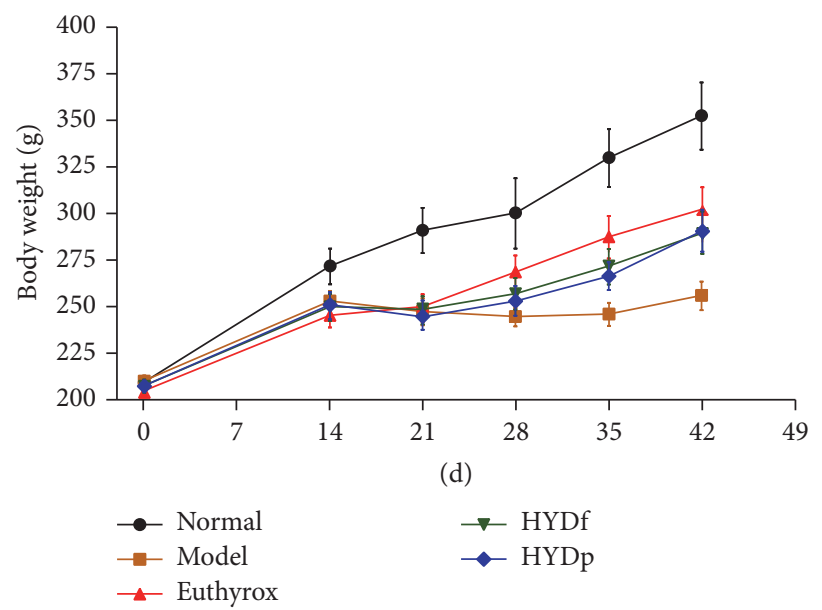

(a)

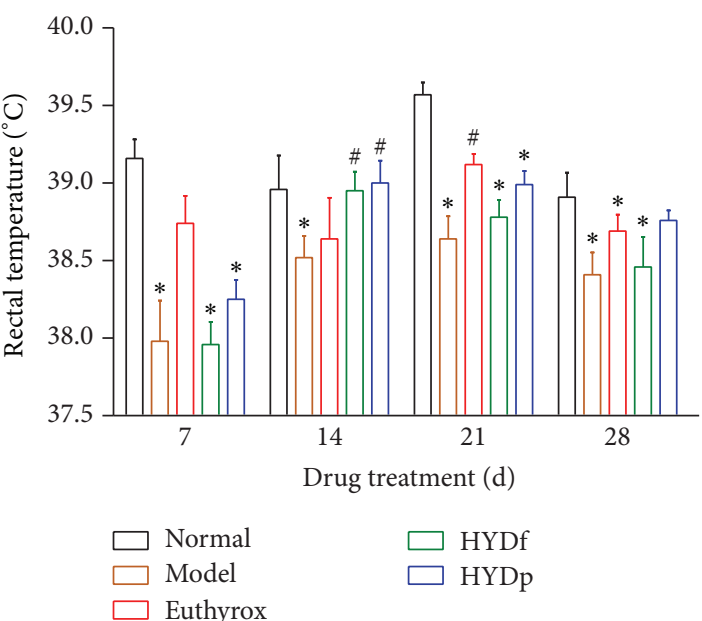

(b)

FIgURE 1: (a) Mean body weight changes in PTU-induced goiter rats. (b) Temperature changes in PTU-induced goiter rats after 28-day drug treatment. Data are presented as mean $\pm \mathrm{SD},{ }^{*} P<0.05$ versus normal; ${ }^{\#} P<0.05$ versus model.

However, no difference was observed on body weight change in HYDf and HYDp.

After drug treatment for 28 days, there were obvious decreases of rectal temperature in the model, Euthyrox, and HYDf treatment groups compared to the normal group $(P<$ $0.05)$. The rectal temperature of HYDp treatment group was statistically higher than that of HYDf treatment group $(P<$ $0.05)$.

3.2. Comparison of Relative Thyroid Weights and Serum Levels of FT3, FT4, TSH, and TRH. Relative thyroid weights of model group were significantly increased $(P<0.01)$, its serum FT4 levels were significantly lower than those of the normal controls $(P<0.01)$, and serum TSH levels were higher than those of the normal controls $(P<0.05)$, indicating the successful production of PTU-induced hypothyroidism goiter model (Figure 2).

Euthyrox, HYDf, and HYDp showed a significant effect on the PTU-induced goiter rats after 28-day treatment (Figure 2(a)). In addition, thyroid enlargement was suppressed significantly in HYDf and HYDp treatment groups compared to Euthyrox group $(P<0.01)$.

The reduced levels of FT4 in the serum of goiter rats could be increased by Euthyrox, HYDf, and HYDp $(P<$ $0.01 ; P<0.05$, Figure 2(c)). In addition, the elevated serum levels of TSH and TRH in goiter rats were decreased by their treatments $(P<0.05$, Figures $2(\mathrm{~d})$ and $2(\mathrm{e}))$.

\subsection{Comparison of Some Liver/Renal Function Parameters.} Compared to the normal controls, relative liver/kidney weights of model group were decreased $(P<0.05$, Figure 3$)$, and its serum activities of AST and Cr were obvious higher $(P<0.05)$; serum UA and $\mathrm{Cr}$ levels of Euthyrox treatment group were higher $(P<0.01 ; P<0.05)$; relative liver/kidney weights of the HYDf treatment group were decreased $(P<$ $0.05)$, and its serum UA and Cr levels were higher $(P<0.05)$.
The HYDp treatment group significantly improved the relative renal weights as well as the activities of AST, ALP, and Cr compared to the goiter model group $(P<0.01)$.

Besides, compared to the HYDf treatment group, the HYDp treatment group significantly improved the relative renal weights and the serum levels of AST, ALP, UA, and $\mathrm{Cr}$ $(P<0.01)$.

3.4. Histological Observations of the Thyroid Gland in Different Groups. Structurally, thyroid gland in PTU-induced goiter model group showed a diffuse homogeneous pattern of follicular epithelial cells hyperplasia with limited volumes of stored colloid compared to normal control group (Figure 4). Although the physiological parameters had returned to normal values, thyroid gland in the Euthyrox treatment group did not return to a morphologically euthyroid state (Figure 4(c)), which was consistent with the results of relative thyroid weights assessment. In contrast, follicular cell hyperplasia of the thyroid gland was obviously improved by HYDf and HYDp treatment (Figures 4(d) and 4(e)); no remarkable differences were found between the two groups.

3.5. $\mathrm{Tg} m R N A$ and TPO mRNA Levels of Different Groups. RT-PCR analysis showed that the mRNA levels of Tg and TPO expression in thyroid tissues of the goiter model group were both higher than those in normal group $(P<0.05)$. The administration of HYDf and HYDp could markedly decrease TPO expression compared to model group $(P<$ 0.05 , Figure 5).

\section{Discussion}

Haizao Yuhu Decoction has been widely used to treat thyroid-related diseases especially goiter with few side effects in TCM. Although the mechanisms are not clear so far, it is considered as a multistep process involving thyroid hormone 


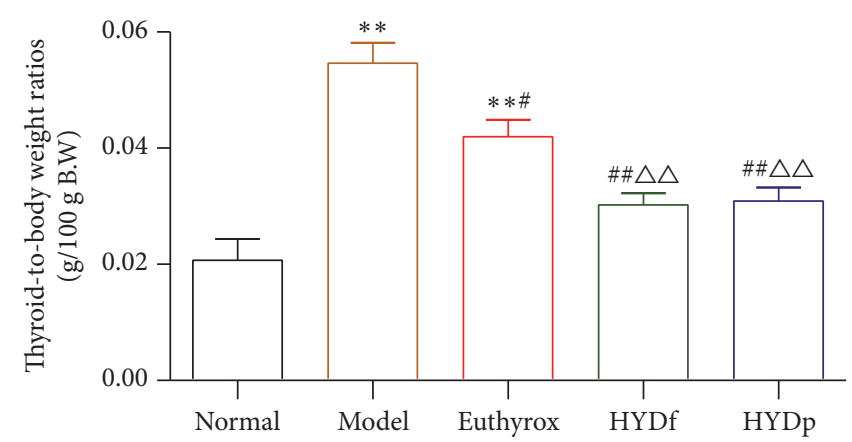

(a)

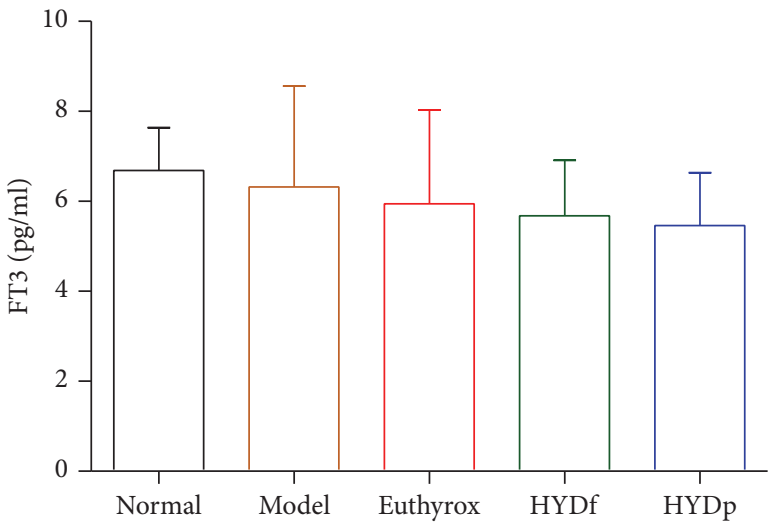

(b)

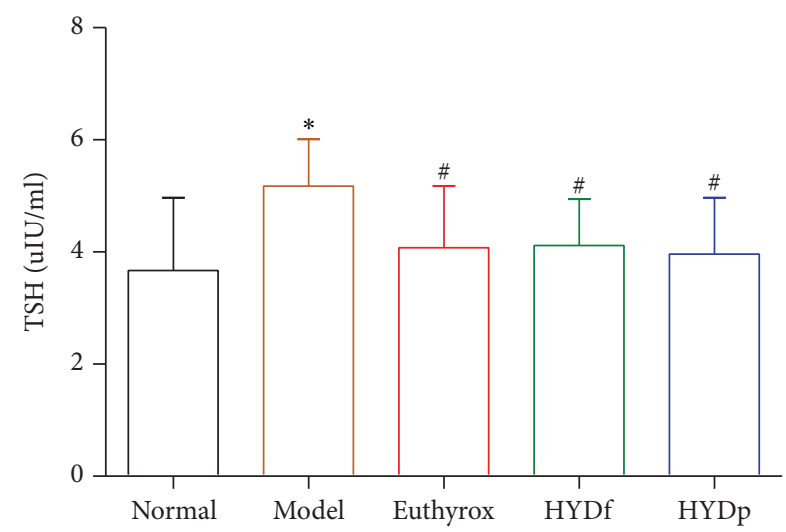

(d)

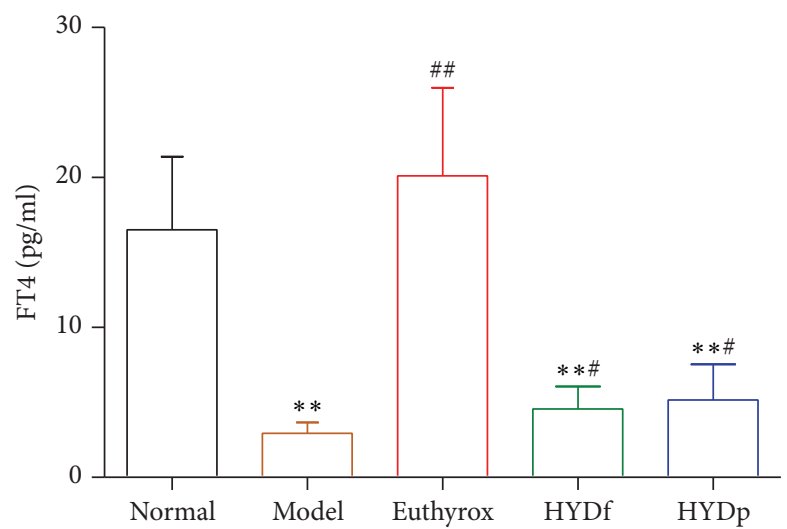

(c)

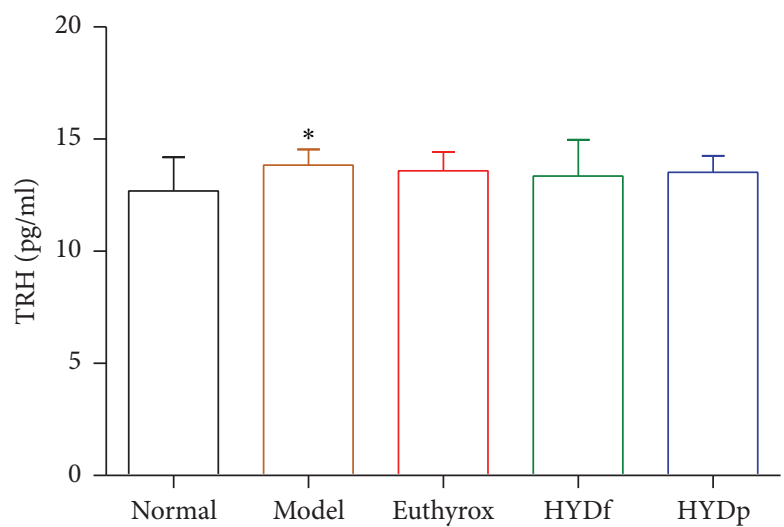

(e)

Figure 2: Comparison of relative thyroid weights (a), serum levels of FT3 (b), FT4 (c), TSH (d), and TRH (e). (a) Thyroid enlargement of goiter rats could be significantly suppressed by Euthyrox, HYDf, and HYDp treatment. HYDf and HYDp showed significant effect on the PTU-induced goiter rats, which were better than that of positive control Euthyrox. (b) No difference was observed on serum levels of FT3 change in different treatment groups. (c) The reduced serum levels of FT4 in goiter rats were increased in Euthyrox, HYDf, and HYDp treatment groups. (d), (e) The elevated serum levels of TSH and TRH in goiter rats were decreased by their treatment. Data are represented as the mean $\pm \mathrm{SD},{ }^{*} P<0.05$ and ${ }^{* *} P<0.01$ versus normal; ${ }^{\#} P<0.05$ and ${ }^{\# \#} P<0.01$ versus model; ${ }^{\triangle \triangle} P<0.01$ versus Euthyrox.

synthesis, antioxidative stress, and immunomodulatory effect $[14,16]$. Notably, herb pair HZ-GC, as one of "eighteen antagonistic medicaments" which means that the two herbs should not be used in the same prescriptions in order to avoid mutually, has been demonstrated regulating targets into the synthesis of thyroid hormone [16], indicating the critical roles of this herb pair in HYD acting on goiter. The two different species of Sargassum, Sf and Sp, were studied for their pharmacological effects and acute toxicity [12]. The results of this initial study clearly demonstrated that there were marked differences in their chemical components and acute toxicity.

Therefore, we speculated that the use of different species of Sargassum in formula compound HYD (HYDf and HYDp) could have different pharmacological effects, accordingly, as for herb pair HZ-GC, the use of different species of 


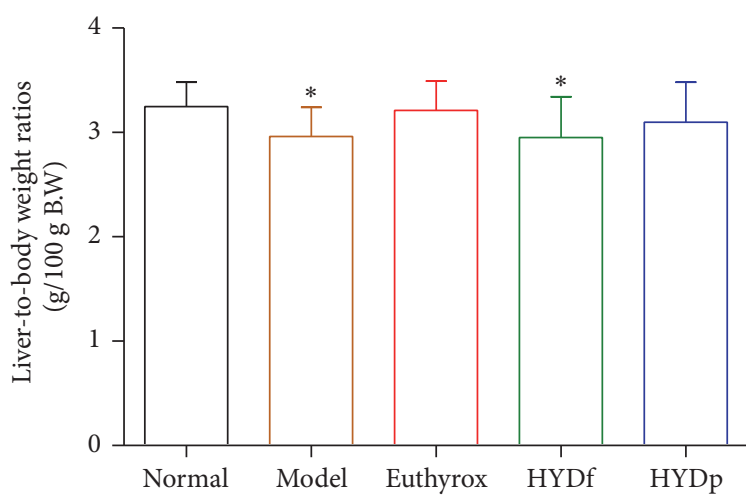

(a)

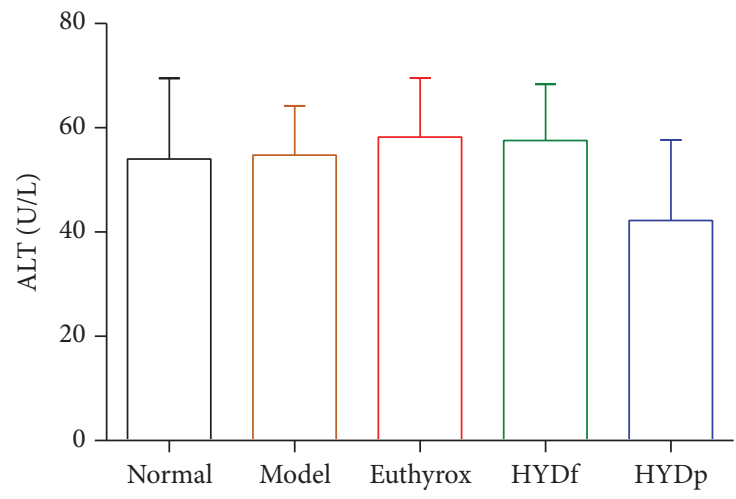

(c)

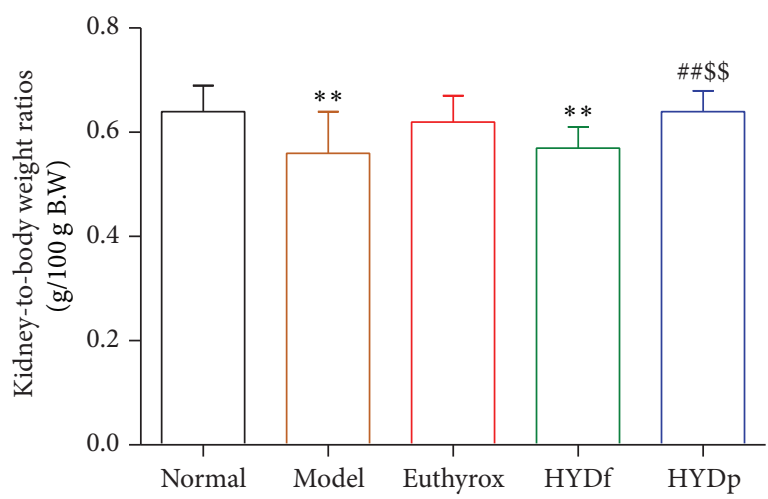

(e)

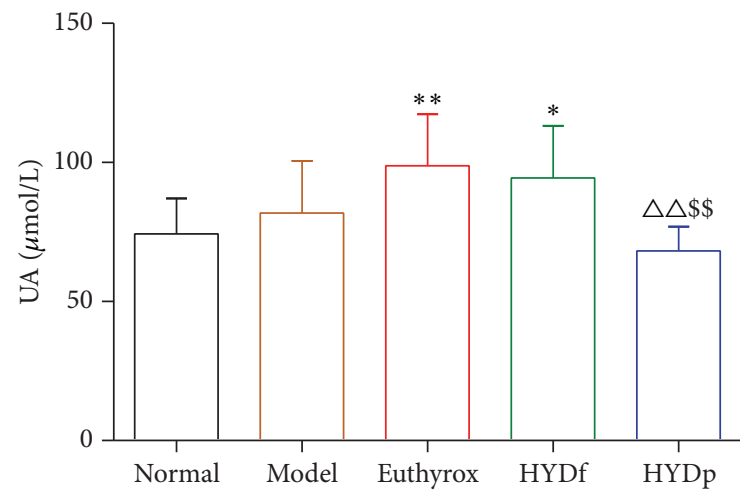

(g)

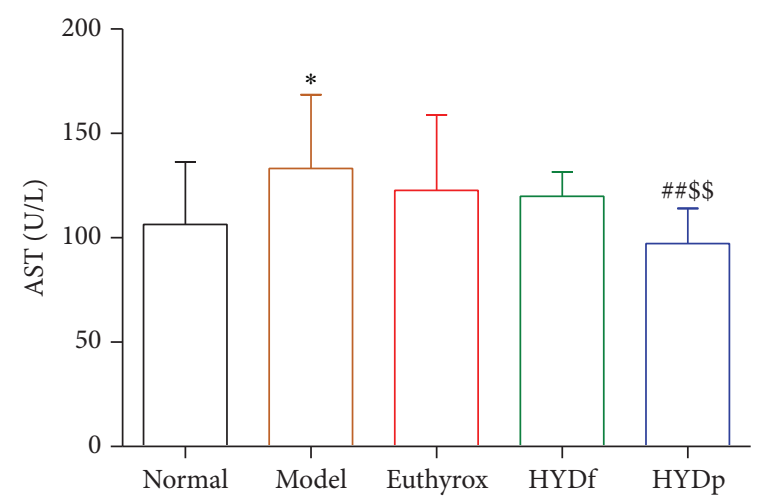

(b)

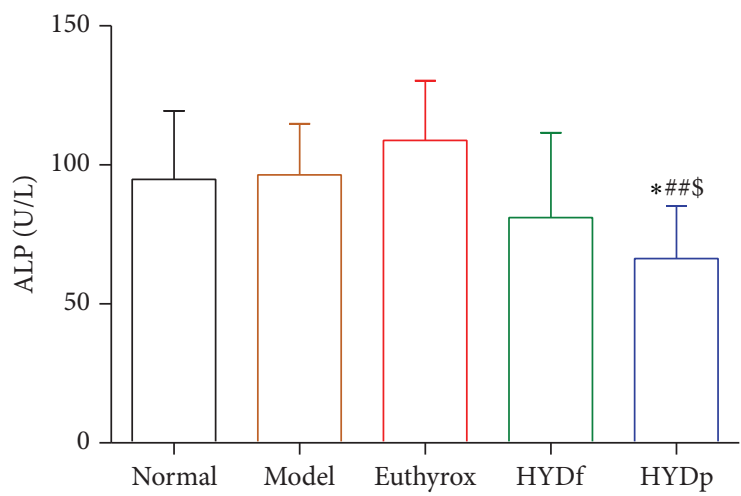

(d)

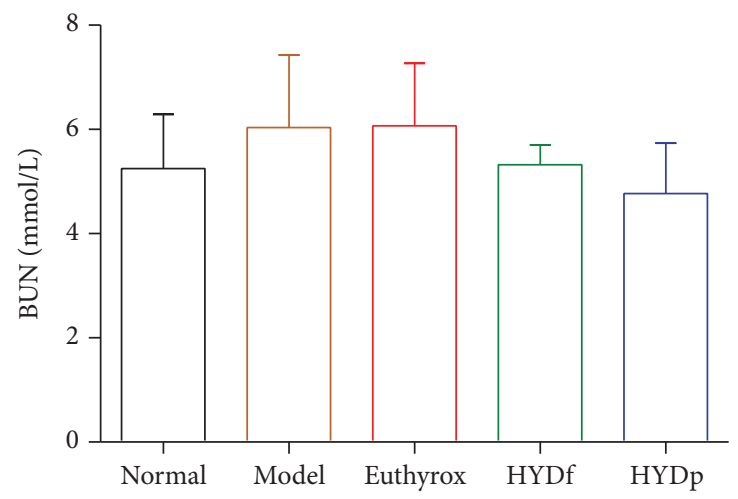

(f)

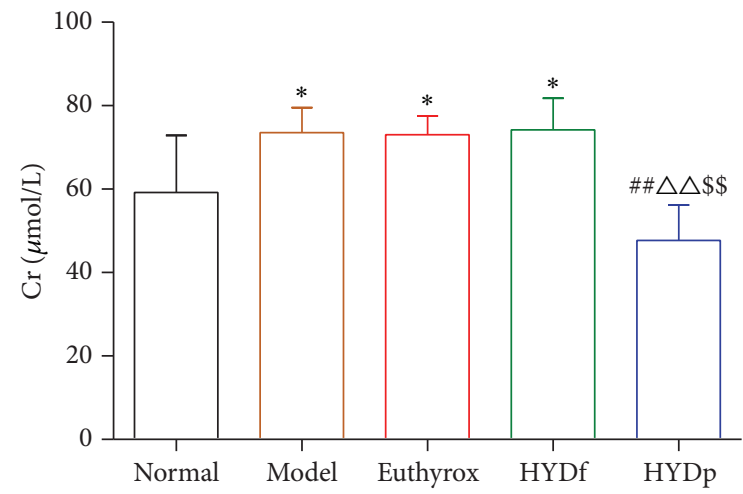

(h)

FIGURE 3: Comparison of relative liver weights (a), serum activities of AST (b), ALT (c), ALP (d), relative kidney weights (e), serum levels of $\operatorname{BUN}(\mathrm{f}), \mathrm{UA}(\mathrm{g})$, and $\mathrm{Cr}(\mathrm{h})$. Data are presented as mean $\pm \mathrm{SD},{ }^{*} P<0.05$ and ${ }^{* *} P<0.01$ versus normal; ${ }^{\# \#} P<0.01$ versus modle; ${ }^{\triangle \triangle} P<0.01$ versus Euthyrox; ${ }^{\$} P<0.05$ and ${ }^{\$} P<0.01$ versus HYDf. 


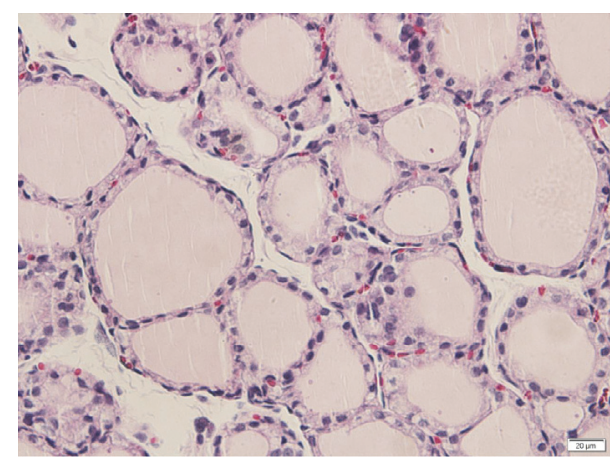

(a)

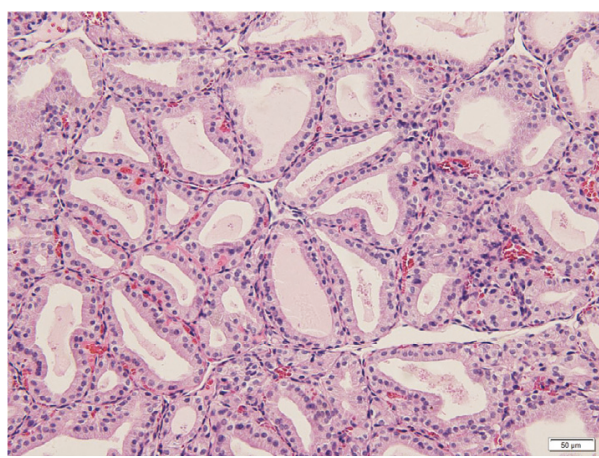

(c)

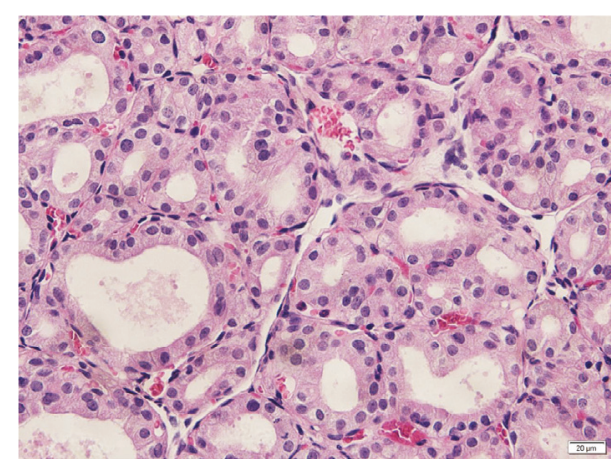

(b)

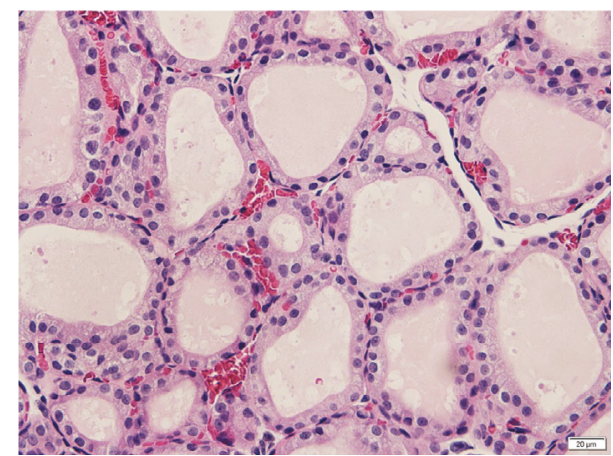

(d)

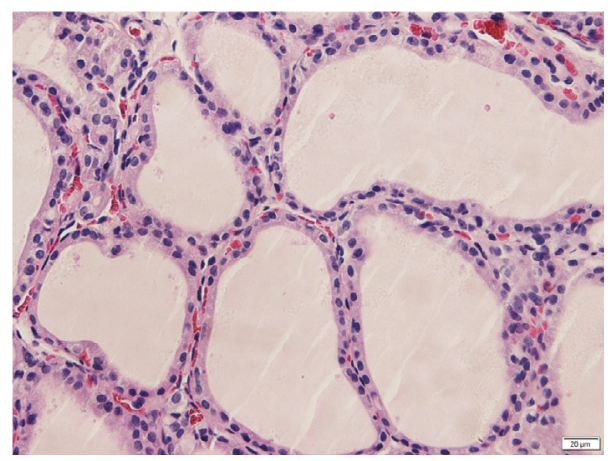

(e)

Figure 4: Histology of thyroid gland (H-E staining 400x). (a) Normal control. (b) Goiter model. (c) Goiter rats treated with Euthyrox. (d) Goiter rats treated with HYDf. (e) Goiter rats treated with HYDp.

Sargassum, herb pair Sf-GC, and herb pair Sp-GC could show different efficacy and toxicity, which might relate to the antagonistic medicament. To investigate whether the use of different species of Sargassum is related to antagonistic medicament, we proposed the present study to systematically evaluate antigoitrous effect (FT3, FT4, thyroid weight, and histological changes) and toxicity (liver/renal function parameters) and to clarify the potential underlying mechanism (TSH, TRH, Tg mRNA, and TPO mRNA) of the two different species of Sargassum in HYD (HYDf and HYDp) in PTU-reduced goiter rats.

This study showed that PTU-induced marked decreases in body weight and rectal temperature with rough hair, slow response, and impaired liver function in goiter rats, with the characteristic of thyroid enlargement and hypothyroidism.
Positive control Euthyrox treatment of 28 days altered the disordered thyroid hormone of hypothyroidism but showed no antigoitrous effects accompanied with impaired renal function. In contrast, both HYDf and HYDp treatment showed antigoitrous effects by inhibition of follicular cell hyperplasia and altering the disordered thyroid hormone of hypothyroidism. However, HYDf treatment displayed liver and renal injury, whereas HYDp treatment could protect the liver and renal function.

TSH and FT4 constitute most standard thyroid function tests and play a central role in the process of diagnosis and treatment of thyroid diseases such as hypothyroidism and thyrotoxicosis [17]. The thyroid gland plays a crucial role, through thyroid hormones (TH) synthesis, in the regulation of various physiological processes, including growth, 


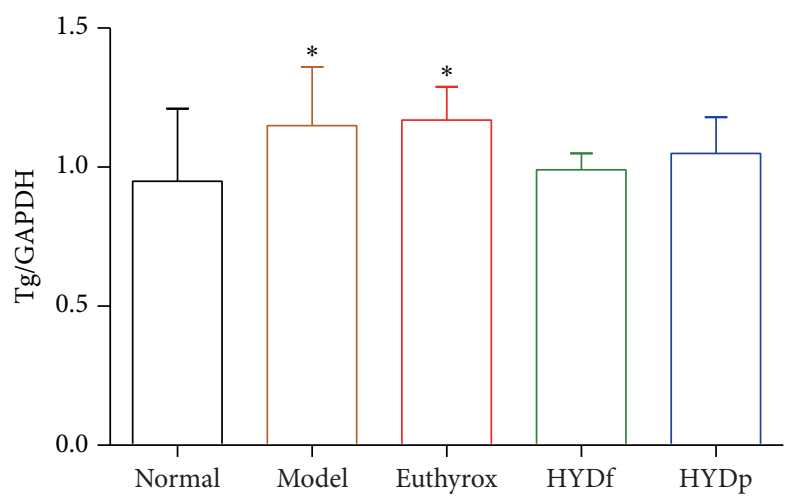

(a)

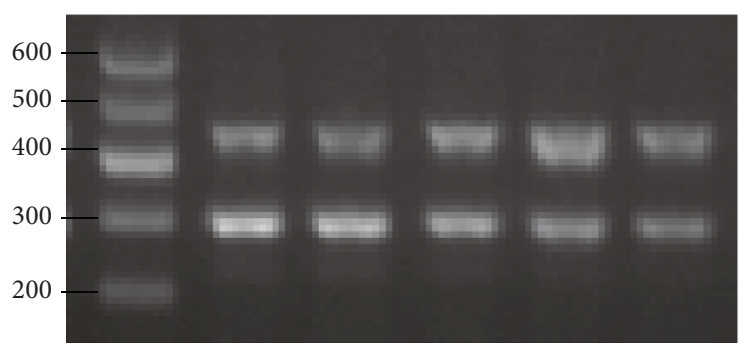

(c)

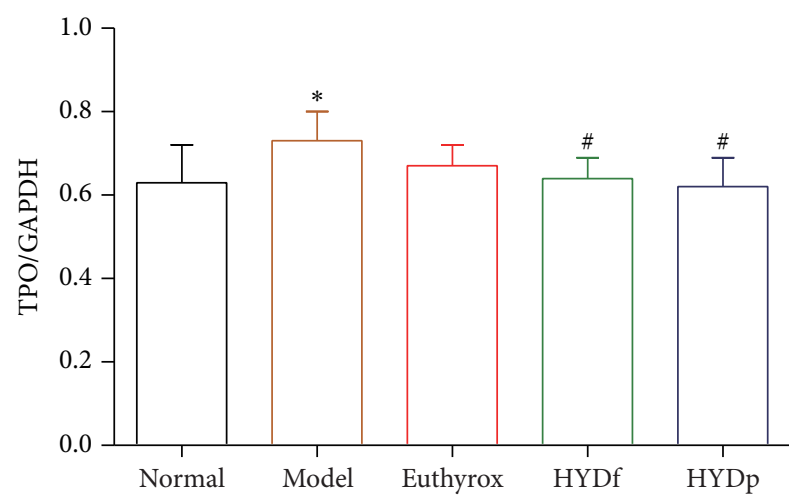

(b)

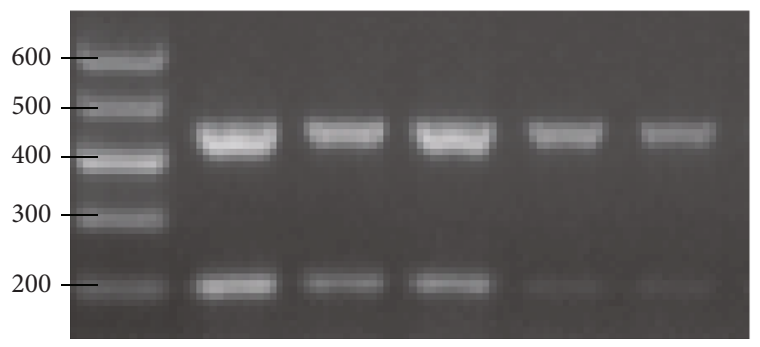

(d)

Figure 5: Tg mRNA (a, c) and TPO mRNA (b, d) levels of different groups. Data are presented as mean \pm SD. ${ }^{*} P<0.05$ versus normal; ${ }^{\#} P<0.05$ versus model.

development, and metabolic stability. The synthesis of TH is regulated by hypothalamus-pituitary-thyroid (HPT) axis in the negative feedback loop [18]. The HPT axis constitutes the main pathogenesis of goiter, with TSH being the strongest stimulatory factor for thyroid hyperplasia and proliferation in thyroid development, growth, and function. TSH can induce the expression and activate the critical genes involved in thyroid hormone formation sodium iodide symporter (NIS), $\mathrm{Tg}$, and TPO [19-21]. The initial precursor of TH is Tg. TPO catalyzes the coupling of these iodotyrosine residues to form TH [22]. It was shown in the rat thyroid that Tg stored in the follicular lumen was a potent regulator of thyroid-specific gene expression to maintain the function of individual follicles [23]. Variants in the Tg gene or TPO gene have been linked to goiter, autoimmune thyroid diseases, and thyroid cancer [24-29].

In PTU-reduced goiter rats, TH levels are reduced and TRH levels are elevated. The TRH stimulates the pituitary to produce TSH, which in turn stimulates the thyroid gland to produce thyroid hormones [30] and induces the expression of TPO and Tg gene, and eventually leads to thyroid hyperplasia and proliferation. HYDf and HYDp could exhibit antigoitrous effects by regulating TH levels through alterations in HPT axis, reducing the evaluated TSH level. TSH plays a central role in promoting thyroid cell proliferation involved in the progress of thyroid growth [31]. The decreased TSH could inhibit the expression of TPO gene, one of the causes of thyroid dyshormonogenesis leading to hypothyroidism characterized by normal synthesis of TH and aberrant protein expression as reported $[32,33]$.
The decrease in relative organ weights is an indication of cell constriction [34] implying that PTU and HYDf could cause cellular constriction in both liver and kidney, whereas HYDp could not produce any effect on relative liver/kidney weights investigated.

The transaminases (AST and ALT) are well-known enzymes used as biomarkers to predict possible toxicity to the liver [35]. The elevation of AST as observed in the model control suggested possible hepatic injury. Administration of HYDp lowered the elevated activities of AST comparable to the model control and indicated the liver protection effect of HYDp. Renal function indices such as serum urea, creatinine, and uric acid can be employed to assess the toxic effects of chemicals on the kidney [36]. The elevations of the indices as observed in the model, Euthyrox, and HYDf treatment rats suggested possible renal injury; HYDp showed the nontoxic and kidney protection effect.

In summary, this study demonstrated that both HYDf and HYDp could exhibit antigoitrous effect through alterations in HPT axis and inhibition of the TPO gene expression; there was no difference in the antigoitrous effects between the two different species of Sargassum application in HYD. HYDp showed the nontoxic and liver/kidney protection effect, but HYDf could cause cellular constriction in both liver and kidney. The reason is unclear and further analysis is needed to clarify.

There are few published reports of experimental studies of antagonistic medicament herb pair HZ-GC; this study has evaluated the safety and efficacy of its application in HYD in goiter rats at the molecular, cellular, and whole 
level and compared the two species of Sargassum further. We provide a reliable way to clarify the possible mechanism of the antagonistic medicament herb pair HZ-GC for its application. The research on the potential mechanism is underway in our laboratory and will be reported shortly.

\section{Conflicts of Interest}

The authors declare that they have no conflicts of interest.

\section{Acknowledgments}

This study was supported by the National Natural Science Foundation of China (81573630).

\section{References}

[1] L. Hegedüs, S. J. Bonnema, and F. N. Bennedbæk, "Management of simple nodular goiter: current status and future perspectives," Endocrine Reviews, vol. 24, no. 1, pp. 102-132, 2003.

[2] T. H. Brix, K. O. Kyvik, K. Christensen, and L. Hegedüs, "Evidence for a major role of heredity in Graves' disease: a population-based study of two Danish twin cohorts," Journal of Clinical Endocrinology and Metabolism, vol. 86, no. 2, pp. 930934, 2001.

[3] R. M. Ruggeri, S. Sciacchitano, A. Vitale et al., "Serum hepatocyte growth factor is increased in Hashimoto's thyroiditis whether or not it is associated with nodular goiter as compared with healthy non-goitrous individuals," Journal of Endocrinological Investigation, vol. 32, no. 5, pp. 465-469, 2009.

[4] M. Knobel, "Etiopathology, clinical features, and treatment of diffuse and multinodular nontoxic goiters," Journal of Endocrinological Investigation, vol. 39, no. 4, pp. 357-373, 2016.

[5] H. Yamashita, S. Noguchi, S. Watanabe et al., "Thyroid cancer associated with adenomatous goiter: an analysis of the incidence and clinical factors," Surgery Today, vol. 27, no. 6, pp. 495-499, 1997.

[6] B. Biondi and D. S. Cooper, "The clinical significance of subclinical thyroid dysfunction," Endocrine Reviews, vol. 29, no. 1, pp. 76-131, 2008.

[7] M. N. Andersen, A.-M. S. Olsen, J. C. Madsen et al., "Levothyroxine substitution in patients with subclinical hypothyroidism and the risk of myocardial infarction and mortality," PLOS ONE, vol. 10, no. 6, Article ID e0129793, 2015.

[8] V. Fatourechi, "Mild thyroid failure [subclinical hypothyroidism]: to treat or not to treat?" Comprehensive Therapy, vol. 28, no. 2, pp. 134-139, 2002.

[9] X.-H. Song, R.-Z. Zan, C.-H. Yu, and F. Wang, "Effects of modified Haizao Yuhu Decoction in experimental autoimmune thyroiditis rats," Journal of Ethnopharmacology, vol. 135, no. 2, pp. 321-324, 2011.

[10] Y. X. Liu, G. S. Zhong, H. Y. Liu et al., "Therapeutic effect of HaizaoYuhu decoction with/without seaweed and liquorice anti-drug combination on goiter rats in preferred dosage conditions," Science and technology review, vol. 33, pp. 87-91, 2015.

[11] Y. W. Li, G. S. Zhong, Q. Wang et al., "Clinical application analysis of haizaoyuhu decoction with antagonistic medicinal compatibility," Nanjing TCM University, vol. 27, pp. 317-321, 2011.

[12] L. L. Xiu, S. R. Wang, G. S. Zhong et al., "Research on acute toxicity of Sargassum pallidum, Sargassumfusiforme, Radix
Glycyrrhiaze and HaizaoYuhu Decoction in mice," China Journal of Traditional Chinese Medicine and Pharmacy, vol. 31, pp. 1933-1936, 2016.

[13] T. C. Qi and T. S. Gao, "Comparative study on intervention mechanisms of iodine and haizaoyuhu decoction for goiter caused by iodine defciency," Chinese Archives of Traditional Chinese Medicine, vol. 30, pp. 1211-1214, 2012.

[14] Y.-W. Li, X. Yu, G.-S. Zhong et al., "Effect of different compatibility of Sargassum and Glycyrrhizae Radix in Haizao Yuhu Decoction on goiter model rats and its mechanism," Chinese Traditional and Herbal Drugs, vol. 45, no. 21, pp. 3124-3130, 2014.

[15] C. Laezza, G. Mazziotti, L. Fiorentino et al., "HMG-CoA reductase inhibitors inhibit rat propylthiouracil-induced goiter by modulating the ras-MAPK pathway," Journal of Molecular Medicine, vol. 84, no. 11, pp. 967-973, 2006.

[16] Y. Zhang, Y. Li, X. Mao et al., "Thyroid hormone synthesis: a potential target of a Chinese herbal formula Haizao Yuhu Decoction acting on iodine-deficient goiter," Oncotarget, vol. 7, no. 32, pp. 51699-51712, 2016.

[17] R. W. V. Flynn, T. M. MacDonald, A. D. Morris, R. T. Jung, and G. P. Leese, "The thyroid epidemiology, audit, and research study: thyroid dysfunction in the general population," Journal of Clinical Endocrinology and Metabolism, vol. 89, no. 8, pp. 38793884, 2004.

[18] M. Nilsson, "Molecular and cellular mechanisms of transepithelial iodide transport in the thyroid," BioFactors, vol. 10, no. 2-3, pp. 277-285, 1999.

[19] P. Kopp, "Thyroid hormone synthesis, thyroid iodine metabolism," in In Werner and Ingbar's the thyroid, a fundamental and clinical text, 9thed., L. Braverman, and R. Utiger, Eds., pp. 5276, Lippincott, Williams, Wilkins, Philadelphia, Pa, USA, 9th edition, 2005.

[20] N. S. Halmi, D. K. Granner, D. J. DoughmaN, B. H. Peters, and G. Muller, "Biphasic effect of TSH on thyroidal iodide collection in rats," Endocrinology, vol. 67, no. 1, pp. 70-81, 1960.

[21] M. Nilsson, "Iodide handling by the thyroid epithelial cell," Experimental and Clinical Endocrinology and Diabetes, vol. 109, no. 1, pp. 13-17, 2001.

[22] A. Taurog, M. L. Dorris, and D. R. Doerge, "Mechanism of simultaneous iodination and coupling catalyzed by thyroid peroxidase," Archives of Biochemistry and Biophysics, vol. 330, no. 1, pp. 24-32, 1996.

[23] Y. Ishido, K. Yamazaki, M. Kammori et al., “Thyroglobulin suppresses thyroid-specific gene expression in cultures of normal but not neoplastic human thyroid follicular cells," Journal of Clinical Endocrinology and Metabolism, vol. 99, no. 4, pp. E694E702, 2014.

[24] A. S. Alzahrani, E. Y. Baitei, M. Zou, and Y. Shi, "Metastatic follicular thyroid carcinoma arising from congenital goiter as a result of a novel splice donor site mutation in the thyroglobulin gene," Journal of Clinical Endocrinology and Metabolism, vol. 91, no. 3, pp. 740-746, 2006.

[25] J. Corral, C. Martin, R. Pérez et al., "Thyroglobulin gene point mutation associated with non-endemic simple goitre," The Lancet, vol. 341, no. 8843, pp. 462-464, 1993.

[26] C. Pérez-Centeno, R. González-Sarmiento, M. T. Mories, J. J. Corrales, and J. M. Miralles-García, "Thyroglobulin exon 10 gene point mutation in a patient with endemic goiter," Thyroid, vol. 6, no. 5, pp. 423-427, 1996.

[27] Y. Ban, D. A. Greenberg, E. Concepcion, L. Skrabanek, R. Villanueva, and Y. Tomer, "Amino acid substitutions in the 
thyroglobulin gene are associated with susceptibility to human and murine autoimmune thyroid disease," Proceedings of the National Academy of Sciences of the United States of America, vol. 100, no. 25, pp. 15119-15124, 2003.

[28] A. Hishinuma, S. Fukata, S. Nishiyama et al., "Haplotype analysis reveals founder effects of thyroglobulin gene mutations C1058R and C1977S in Japan," Journal of Clinical Endocrinology and Metabolism, vol. 91, no. 8, pp. 3100-3104, 2006.

[29] A. Hishinuma, S. Fukata, K. Kakudo, Y. Murata, and T. Ieiri, "High incidence of thyroid cancer in long-standing goiters with thyroglobulin mutations," Thyroid, vol. 15, no. 9, pp. 1079-1084, 2005.

[30] A. Gökhan Özgen, G. Keser, N. Erdem et al., "Hypothalamushypophysis-thyroid axis, triidothyronine and antithyroid antibodies in patients with primary and secondary Sjögren's syndrome," Clinical Rheumatology, vol. 20, no. 1, pp. 44-48, 2001.

[31] M. C. Eggo, V. M. Quiney, and S. Campbell, "Local factors regulating growth and function of human thyroid cells in vitro and in vivo," Molecular and Cellular Endocrinology, vol. 213, no. 1, pp. 47-58, 2003.

[32] G. Medeiros-Neto, H. M. Targovnik, and G. Vassart, "Defective thyroglobulin synthesis and secretion causing goiter and hypothyroidism," Endocrine Reviews, vol. 14, pp. 165-183, 1993.

[33] G. Medeiros-Neto, M. Knobel, and L. J. DeGroot, "Genetic disorders of the thyroid hormone synthesis," in Genetics in Endocrinology, J. D. Baxter, Ed., pp. 375-402, Lippincott, Williams, Wilkins, Philadelphia, Pa, USA, 2002.

[34] B. M. Schmidt, N. Ilic, A. Poulev, and I. Raskin, "Toxicological evaluation of a chicory root extract," Food and Chemical Toxicology, vol. 45, no. 7, pp. 1131-1139, 2007.

[35] M. F. Rahman, M. K. Siddiqui, and K. Jamil, "Effects of Vepacide (Azadirachta indica) on aspartate and alanine aminotransferase profiles in a subchronic study with rats," Human and Experimental Toxicology, vol. 20, no. 5, pp. 243-249, 2001.

[36] T. O. Sunmonu and O. B. Oloyede, "Biochemical assessment of the effects of crude oil contaminated catfish (Clarias gariepinus) on the hepatocytes and performance of rat," African Journal of Biochemistry Research, vol. 1, no. 5, pp. 83-89, 2007. 


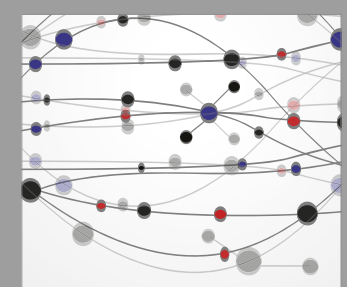

The Scientific World Journal
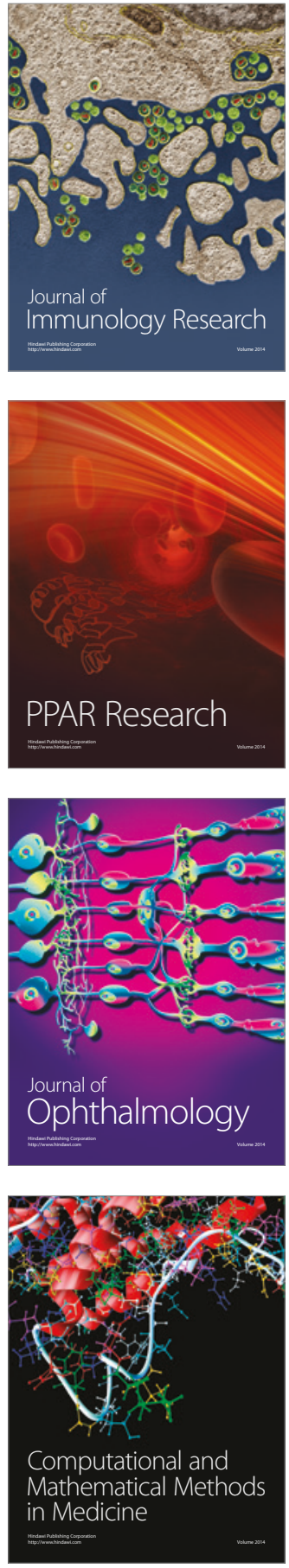

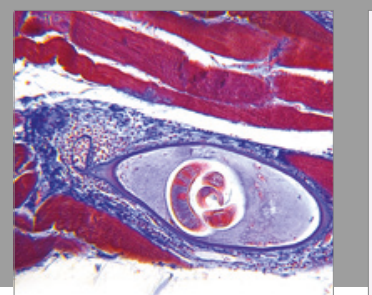

Gastroenterology Research and Practice
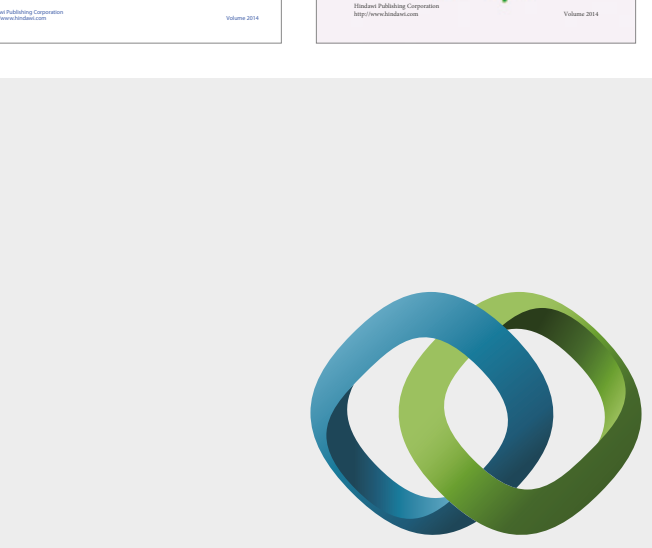

\section{Hindawi}

Submit your manuscripts at

https://www.hindawi.com
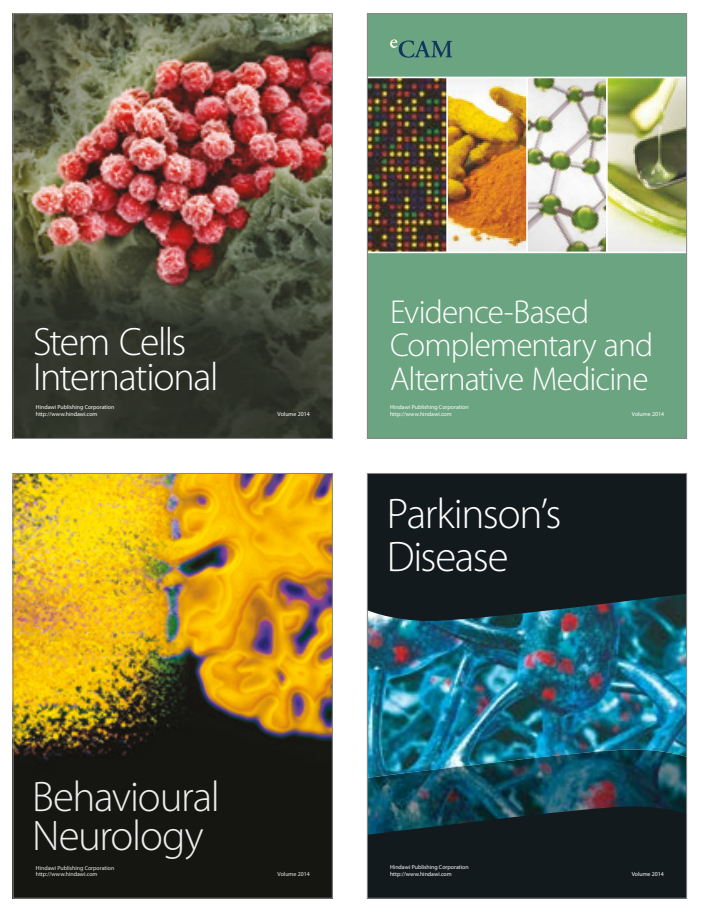
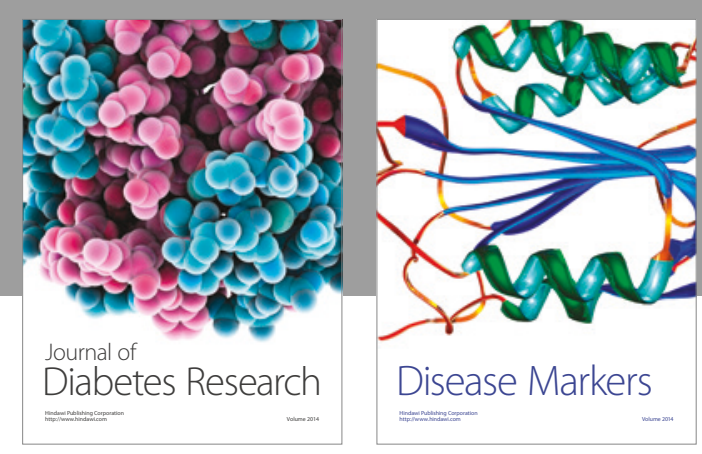

Disease Markers
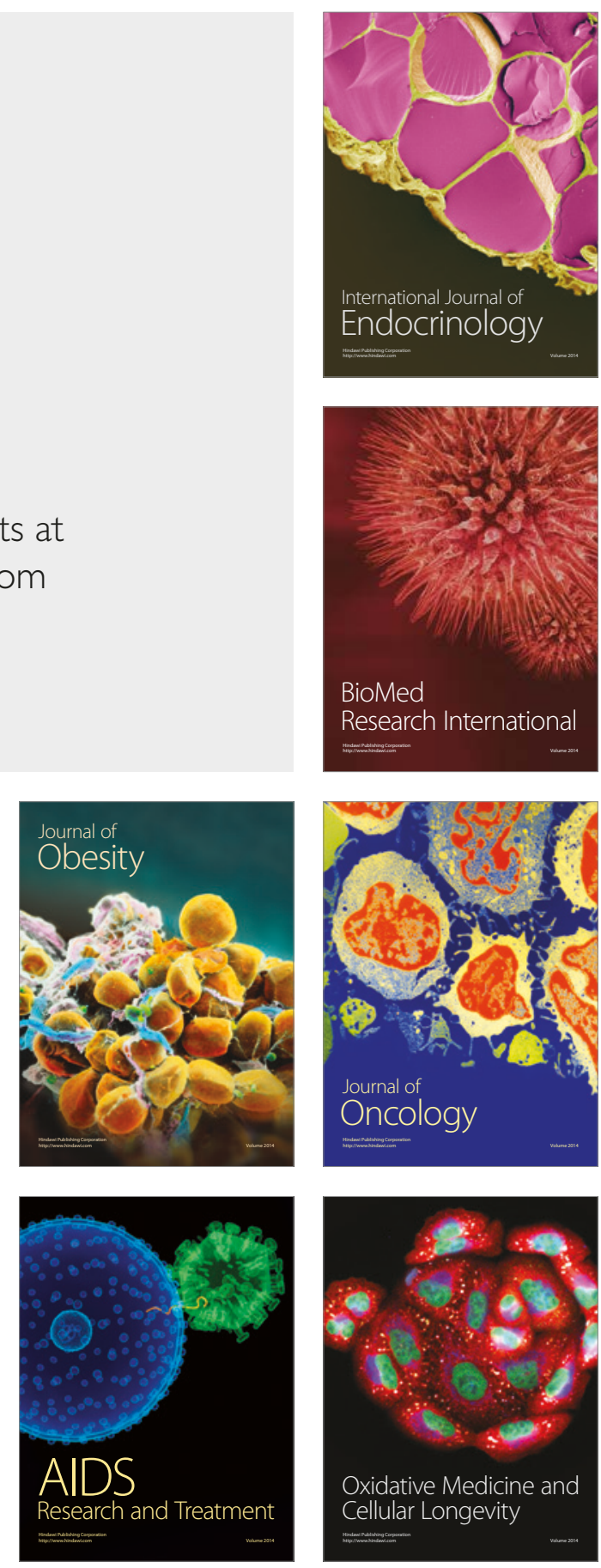